\title{
Physical activity, Sedentary Behaviour and Their Correlates in Adults with Autism Spectrum Disorder: a Systematic Review
}

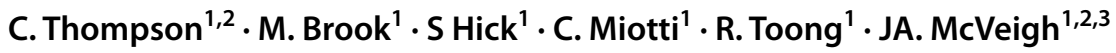

Received: 27 May 2021 / Accepted: 5 February 2022

(c) The Author(s) 2022

\begin{abstract}
The aim of this systematic review was to investigate what is currently known about autistic adults' participation in physical activity and sedentary behaviour and explore any associated correlates of these behaviours. The databases EMBASE, MEDLINE, PsycINFO, PubMed, and SPORTDiscus were searched for relevant literature. The review was registered with PROSPERO (No. 189187). The search identified 2834 papers, with 12 meeting the criteria for inclusion. Correlates of physical activity and/or sedentary behaviour were reported in eight included studies. The limited evidence identified suggest that autistic adults are unlikely to meet Australia's physical activity guidelines. Future research should use device-derived measurement to more accurately quantify activity levels within the autistic adult population.
\end{abstract}

Keywords Autism spectrum disorder $\cdot$ Physical activity $\cdot$ Sedentary behaviour $\cdot$ Adults

\section{Introduction}

Physical activity is defined as bodily movements produced by skeletal muscles requiring energy expenditure (World Health Organization, 2017). An individual's daily physical activity pattern consists of various activities along a continuum of intensities, from sedentary behaviour (e.g. sitting, lying) to moderate and vigorous physical activity (MVPA) (e.g. running, swimming) (Department of Health, 2019). Australia's physical activity and sedentary behaviour guidelines recommend that adults perform sufficient physical activity (i.e. participate in $>150 \mathrm{~min} \mathrm{MVPA} /$ week) and minimise and break up their sedentary behaviour (Department of Health, 2019). Health outcomes associated with sufficient physical activity participation include decreased mortality, reduced risk of developing chronic conditions (e.g. cardiovascular disease) (Lear et al., 2017), maintaining healthy blood pressure and cholesterol levels, preventing

C. Thompson

craig.thompson@curtin.edu.au

1 Curtin School of Allied Health, Curtin University, Perth, Australia

2 enAble Institute, Curtin University, Perth, Australia

3 Movement Physiology Laboratory, School of Physiology, University of Witwatersrand, Johannesburg, South Africa obesity and promoting physical and mental health (Kokkinos, 2012; World Health Organization, 2017). Despite the benefits of sufficient participation in physical activity, approximately half of Australian adults were considered insufficiently active based on self-reports in 2017-2018 (Australian Bureau of Statistics, 2019). The patterns and volumes of physical activity and sedentary behaviour are influenced by a wide variety of factors or correlates, including a diagnosis of autism spectrum disorder (ASD).

Autism spectrum disorder is a lifelong, neurodevelopmental condition characterised by deficits in social communication and restricted, repetitive behaviours and interests (American Psychiatric Association, 2013). It is essential to continue to develop knowledge of ASD across the lifespan due to the condition's lifelong nature, and the unique challenges adulthood presents for autistic individuals (Howlin et al., 2015). Autistic adults are reported to have lower participation in domains of life, including education, employment (Knüppel et al., 2019), social relationships (Tobin et al., 2014), leisure and physical activity compared to neurotypical peers (García-Villamisar \& Dattilo, 2010; Hillier et al., 2020). Autistic adults may also experience motor and social skill deficits, difficulties with sensory processing, poor sleep and restrictive behaviour patterns (Nichols et al., 2018). These characteristics may act as unique correlates limiting autistic adults' involvement in a range of occupational roles and activities, 
including participating in recommended physical activity levels (Menear \& Neumeier, 2015).

The research examining correlates of time spent in physical activity and sedentary behaviour among autistic adults is scant. However, several studies have reported on time spent in physical activity and sedentary behaviour (and the correlates thereof) in autistic children. Autistic children spend more time in sedentary behaviour than neurotypical children (Jones et al., 2017; Must et al., 2014) and have lower levels of physical activity (Jones et al., 2017). Access to an outdoor environment, social interactions and being of male gender demonstrated to positively influence physical activity participation in autistic children (Healy \& Garcia, 2019; Pan et al., 2011). A larger number of close friends and lower parental rating of prosocial behaviour negatively impact sedentary behaviour participation in this population (Healy \& Garcia, 2019). Moreover, there are consistent findings that suggest a decline in physical activity participation and lesser adherence to Australia's physical activity and sedentary behaviour guidelines as autistic children age (Healy et al., 2019; Jones et al., 2017; MacDonald et al., 2011; Simpson et al., 2019). It is important to understand the factors impacting upon and health implications of physical activity and sedentary behaviour participation in order to support autistic adults to achieve positive health outcomes (Simpson et al., 2019; Tanguay \& Lohr, 2015).

The usefulness of the evidence for physical activity levels is influenced by the measurement method. Self-report and device-derived measurement are two common methods used to measure physical activity and sedentary behaviour (Yano et al., 2019). Self-report measures of physical activity and sedentary behaviour vary according to a range of factors, including the variables being quantified (e.g. frequency and/or duration of participation), unit of measurement (e.g. length of time, categorical ranking) and method of application (e.g. self-completed questionnaire, interview). The use of measurement devices (i.e. fitness trackers and accelerometers) to quantify physical activity and sedentary behaviour is growing in popularity (Yano et al., 2019). Device-derived measurement of physical activity and sedentary behaviour enables the identification of, and ability to discriminate between characteristics of activity patterns including frequency, duration, type and intensity (Strath et al., 2012).

The current evidence base in autistic children has increasingly used device derived measures of physical activity, as demonstrated by a recent systematic review where 31 of the 35 studies used device derived methods. In the autistic adult population, most studies have used questionnaires, such as the paper by Engelhardt et al. (2017). The limited use of device derived measurement has also meant that studies in the autistic adult population have mainly focused on either physical activity, such as Hamm and Yun (2019), or sedentary behaviours, such as Finke et al. (2018), resulting in a limited understanding of the continuum of activity.

Currently, there is limited evidence examining time spent in physical activity and sedentary behaviour among the autistic adult population, and the evidence regarding correlates of these behaviours is scarce. Therefore, the aim of this review was to systematically investigate what is known about the time that autistic adults spend in physical activity and sedentary behaviour and to explore any associated correlates of these behaviours.

\section{Methods}

\section{Design}

A systematic review of the literature regarding the physical activity and sedentary behaviour prevalence of autistic adults and associated correlates was conducted. The guidelines outlined in the Preferred Reporting Items for Systematic Reviews and Meta-analyses (PRISMA) statement were followed (Moher et al., 2009). Prior to commencing this systematic review, the proposed methodology was registered with PROSPERO (No. 189187) (National Institute for Health Research, 2020).

\section{Search Strategy}

A literature search was conducted using five selected online databases: EMBASE, MEDLINE, PsycINFO, PubMed, and SPORTDiscus. The search strategy included keywords within four categories: (1) population, (2) physical activity, (3) sedentary behaviour and (4) Autism spectrum disorder. Table 1 (insert Table 1 after this paragraph) provides the search terms used for all databases. In order to broaden the scope of the review and ensure all papers with potential for inclusion were captured, no limitations (e.g. year of publication) were applied to initial searches of each respective database. Searches were designed to capture papers published from the establishment of the databases to April 2020.

Papers were included if they were (1) peer-reviewed, (2) written in English, (3) accessible by researchers in full-text and (4) reported a measure of individuals' physical activity and/or sedentary behaviour. Academic theses, commentaries, secondary reviews and papers omitting baseline or habitual physical activity and/or sedentary behaviour data were excluded. In order to further broaden the scope for the review, reference lists of short-listed papers were screened manually to identify any other potentially relevant papers. Following initial database searches and manual review of references, the title and reference of short-listed papers were entered into a shared Microsoft Excel ${ }^{\mathrm{TM}}, 2020$ spreadsheet $^{-}$ for review by all researchers. 
Table 1 Search terms used in all databases

\begin{tabular}{|c|c|c|c|}
\hline \multicolumn{2}{|l|}{ Population } & \multicolumn{2}{|l|}{ Outcome } \\
\hline Age & Diagnosis & Physical activity & Sedentary behaviour \\
\hline $\begin{array}{l}\text { Adult* } \\
\text { Old* adult* } \\
\text { Elder* }\end{array}$ & $\begin{array}{l}\text { Autis* } \\
\text { "Autism spectrum disorder" } \\
\text { ASD } \\
\text { Asperger* } \\
\text { "Autistic disorder" } \\
\text { "Pervasive developmental disorder" } \\
\text { PDDNOS } \\
\text { PDD } \\
\text { "Pervasive developmental disorder not otherwise specified" }\end{array}$ & $\begin{array}{l}\text { "Physical* activ*" } \\
\text { Exercis* } \\
\text { "Motor activit*" } \\
\text { "Locomotor activit*" } \\
\text { Sport* } \\
\text { Acceleromet* } \\
\text { Recreation* }\end{array}$ & $\begin{array}{l}\text { "Sedentary behavio*" } \\
\text { Sedentar* } \\
\text { "Physical inactiv*" } \\
\text { Inactiv* } \\
\text { Gaming } \\
\text { "Television view*" } \\
\text { "TV" } \\
\text { Television } \\
\text { "Video game*" } \\
\text { "Screen time" } \\
\text { "Electronic game* } \\
\text { Computer* } \\
\text { E-game* } \\
\text { Video* } \\
\text { "Screen based media" } \\
\text { "Electronic media" }\end{array}$ \\
\hline
\end{tabular}

A systematic screening process (Figure 1) was conducted to ensure selective inclusion of relevant papers: (1) All four authors screened paper titles and discussed their potential suitability for inclusion; (2) two authors (MB, RT) screened remaining papers' abstracts, with third author (CM) being consulted if a unanimous decision regarding inclusion was not achieved; (3) two authors (MB, RT) screened remaining papers' full text, with a third author (CM) being consulted if a unanimous decision regarding inclusion was not achieved; and (4) all four authors reviewed and agreed upon final papers deemed to have met the inclusion criteria. Authors' individual decisions for each phase of data screening were recorded on a shared Microsoft $\mathrm{Excel}^{\mathrm{TM}}$, 2020 spreadsheet, with individual columns to input ' $N$ ' to represent 'No', ' $\mathrm{Y}$ ' to represent 'Yes' and '?' to represent 'Maybe' next to each respective study.

\section{Quality Assessment}

The Standard Quality Assessment Criteria was used to critically appraise each of the final 12 studies to be included in the review to determine the quality of the studies (Kmet et al., 2004). Four authors completed the quantitative or qualitative checklists for each of the 12 included studies dependent on respective study design (Kmet et al., 2004). To determine the individual study quality, criteria assessed included, but was not exclusive to: study design (e.g. appropriateness), sample selection, size and description (e.g. adequate and representative), random allocation and blinding (e.g. appropriately used), outcome measures, analysis methods (e.g. described and justified), confounding variables (e.g. if they were controlled for) and results (e.g. supporting hypothesis). Each study was awarded a score of '2' for 'Yes', ' 1 ' for 'Partial' or ' 0 ' for 'No' across each criterion on the checklist according to the assessment guidelines (Kmet et al., 2004). For a summary of categorical ratings for each included study, see Appendix A. From the summed categorical ratings, percentage scores were calculated for the quality of each study. All four authors discussed individual scoring and negotiated to full agreement. Studies were then given a categorical rating of quality according to the following protocol: $>80 \%=$ strong, $70-80 \%=$ good, $50-70 \%=$ adequate, $<50 \%=$ limited (Lee et al., 2008). Study quality across the 12 studies was categorically ranked 'good' overall, with an average score of $74 \%$ in accordance with the Standard Quality Assessment Criteria (Table 2) (insert Table 2 at the beginning of page 8) (Kmet et al., 2004).

\section{Data Extraction}

Data regarding participant sample, study design, ASD diagnosis, outcome of interest, instrument employed to collect data, results and correlates were extracted from each of the 12 included studies. The studies were screened by three authors (CM, MB and RT) to complete data extraction independently. To increase accuracy, each study was examined by two of the three screening authors. All data were manually extracted and entered into a shared Microsoft Excel $^{\mathrm{TM}}, 2020$ spreadsheet. Following initial data extraction, information recorded for all studies was manually reviewed and re-assessed by a fourth author ( $\mathrm{SH})$. Following the final review, all authors negotiated the $5 \%$ discrepancy to reach full agreement.

\section{Data Standardisation}

In the 12 included studies, physical activity and sedentary behaviour data were reported on in various formats 


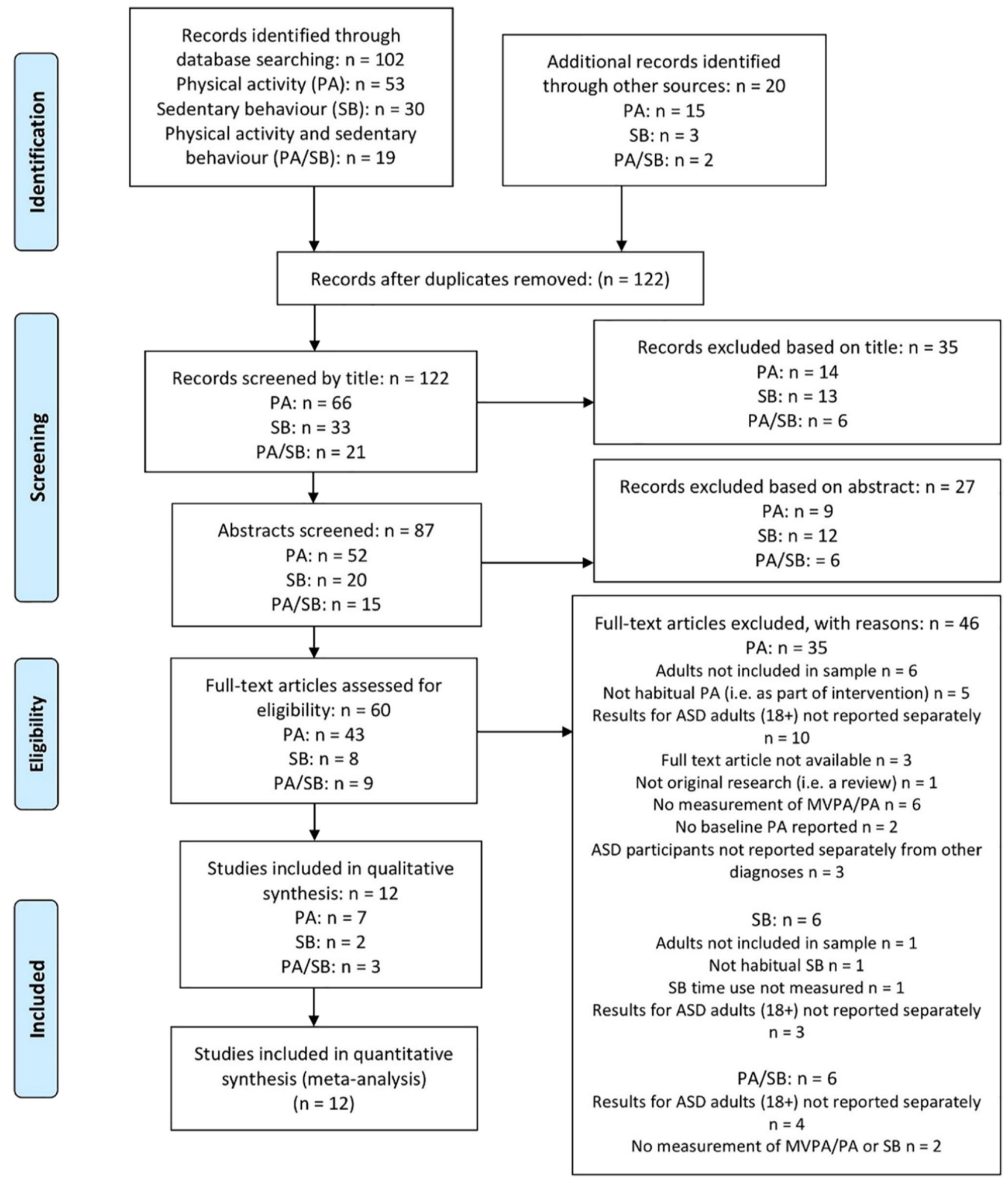

Fig. 1 PRISMA flow diagram of search results

dependent on the study design and outcomes of interest. Prevalence of physical activity was reported as MVPA minutes per day, number of steps per day, MET minutes per week and Godin-Shephard Leisure-Time Physical Activity Questionnaire (GSLTPAQ) scores or self-reported activity level (e.g. number of participations per week). For sedentary 


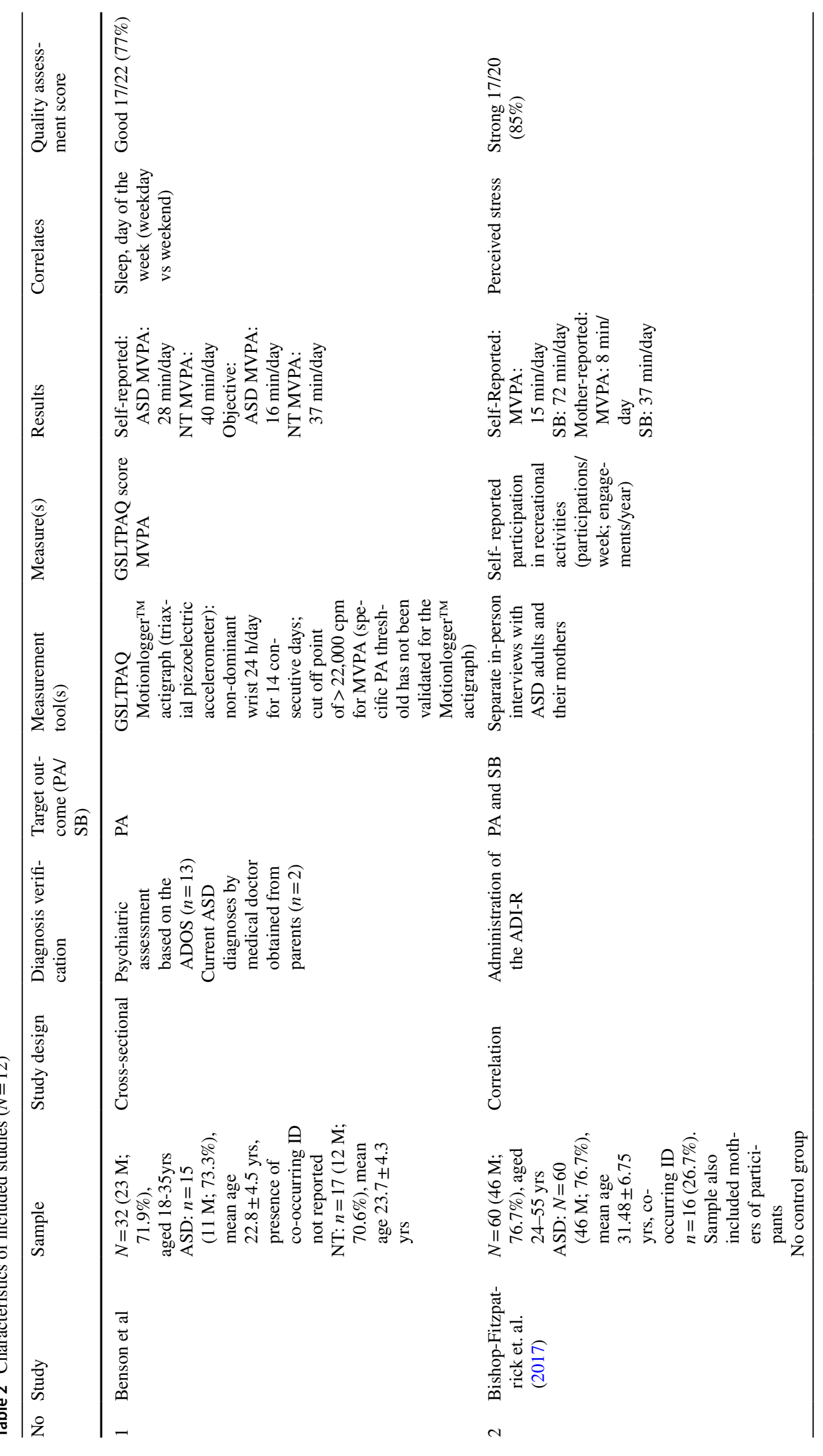




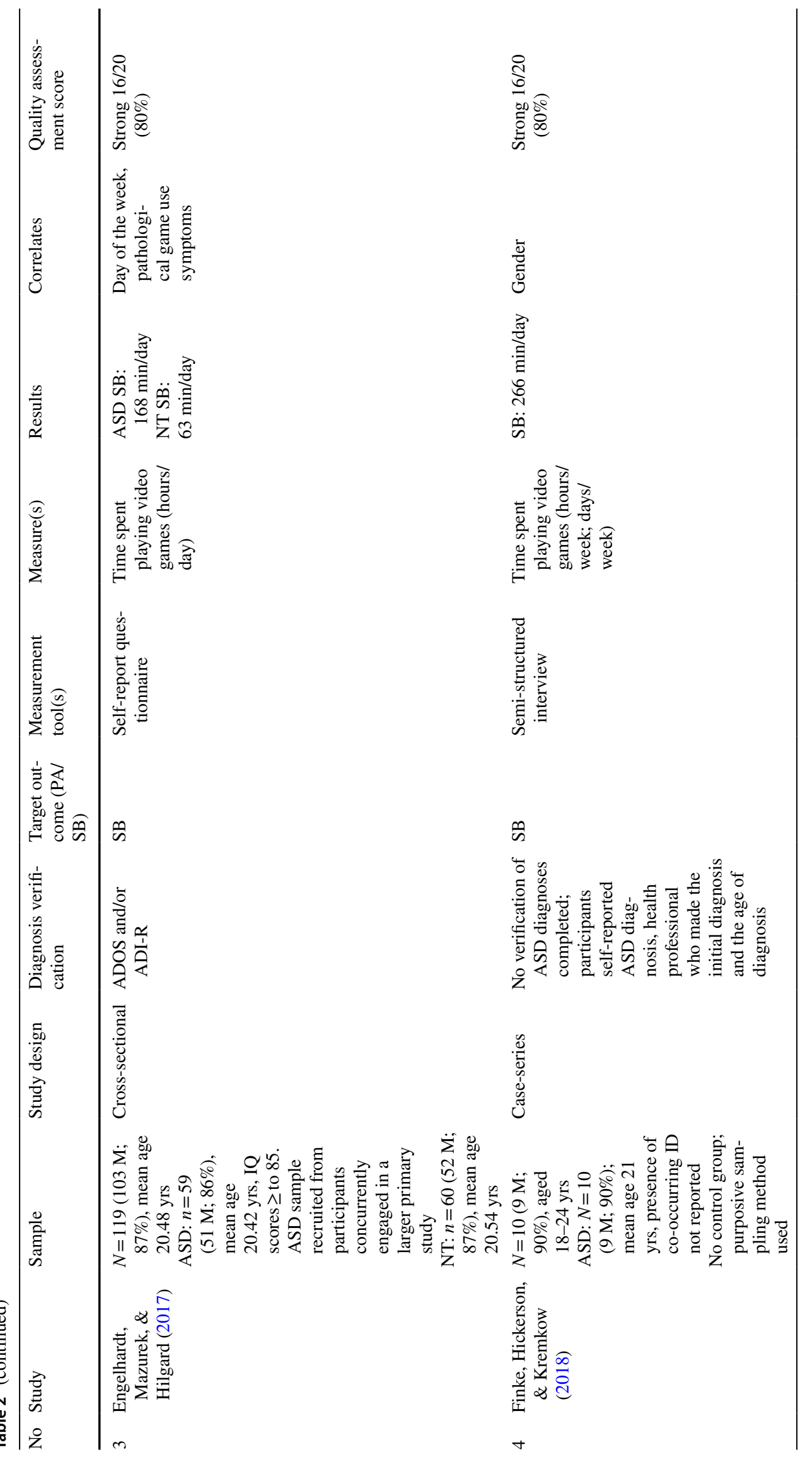




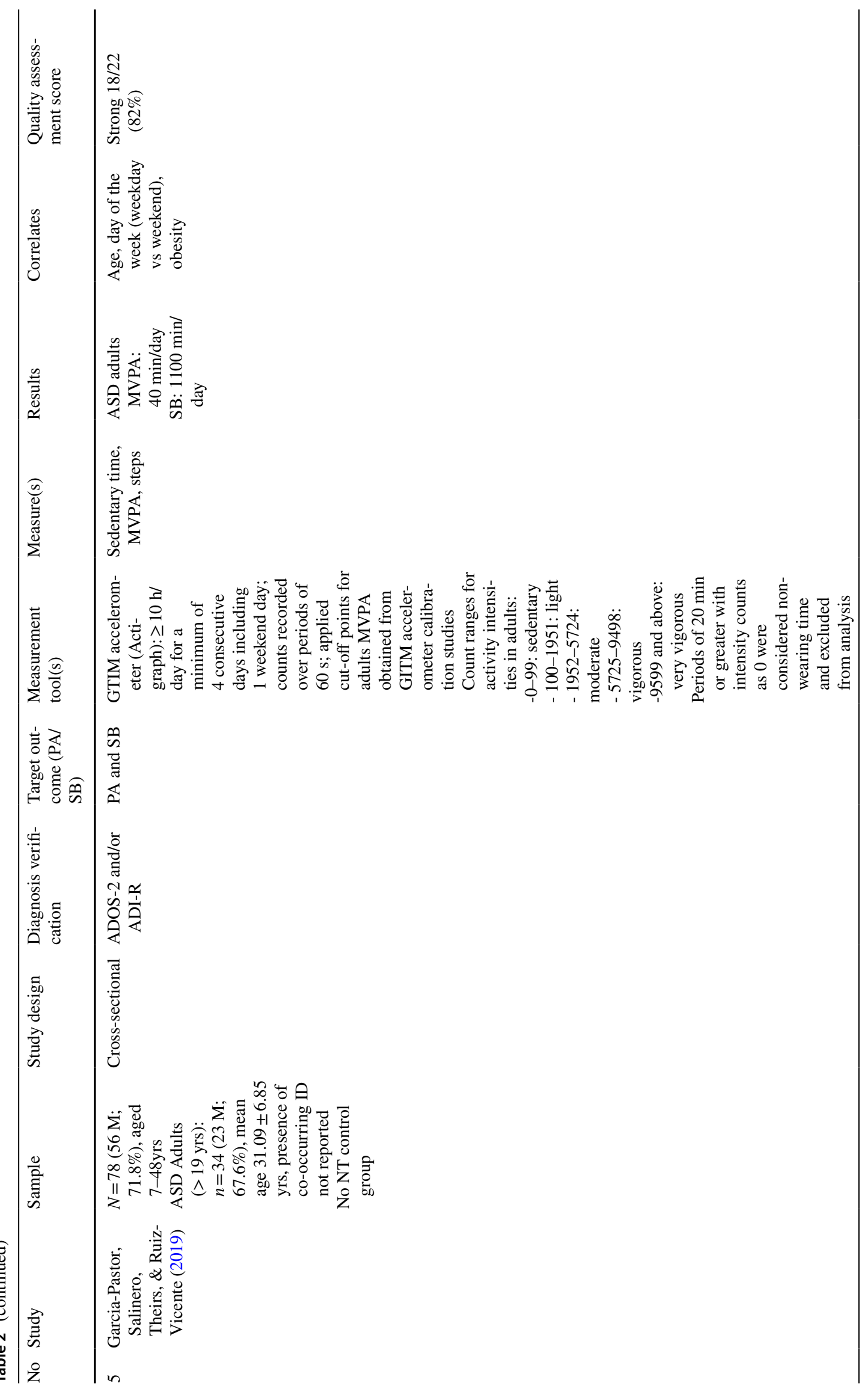




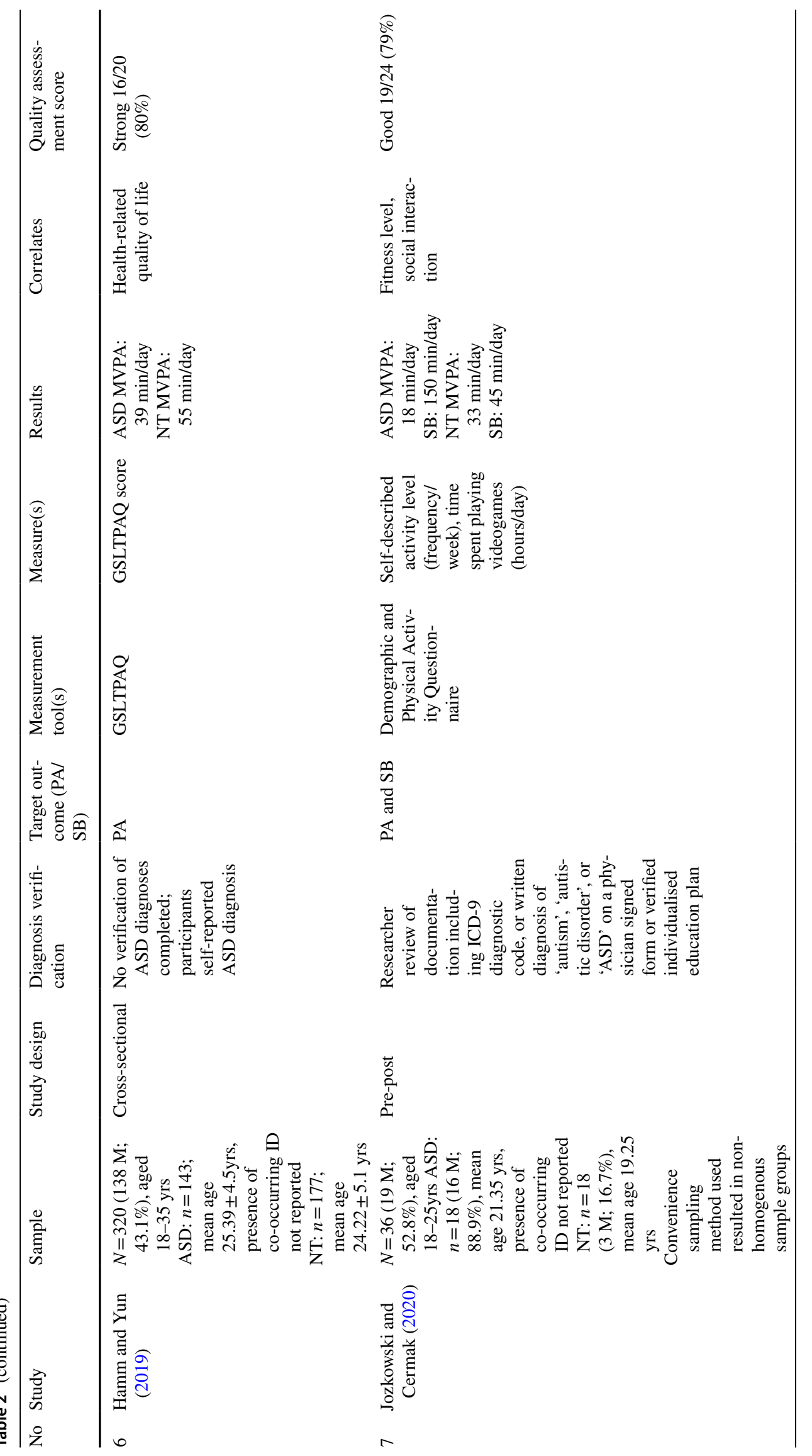




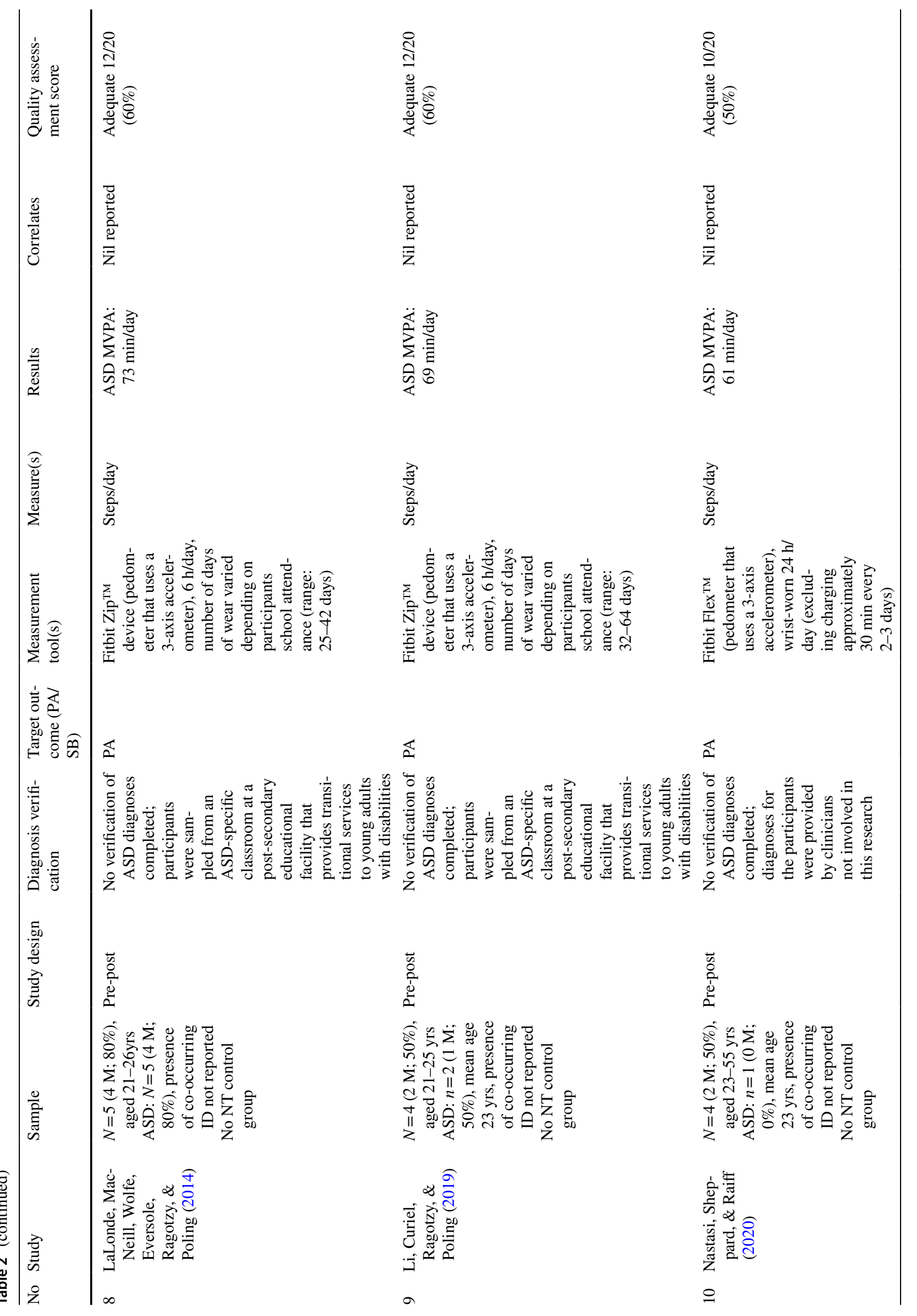




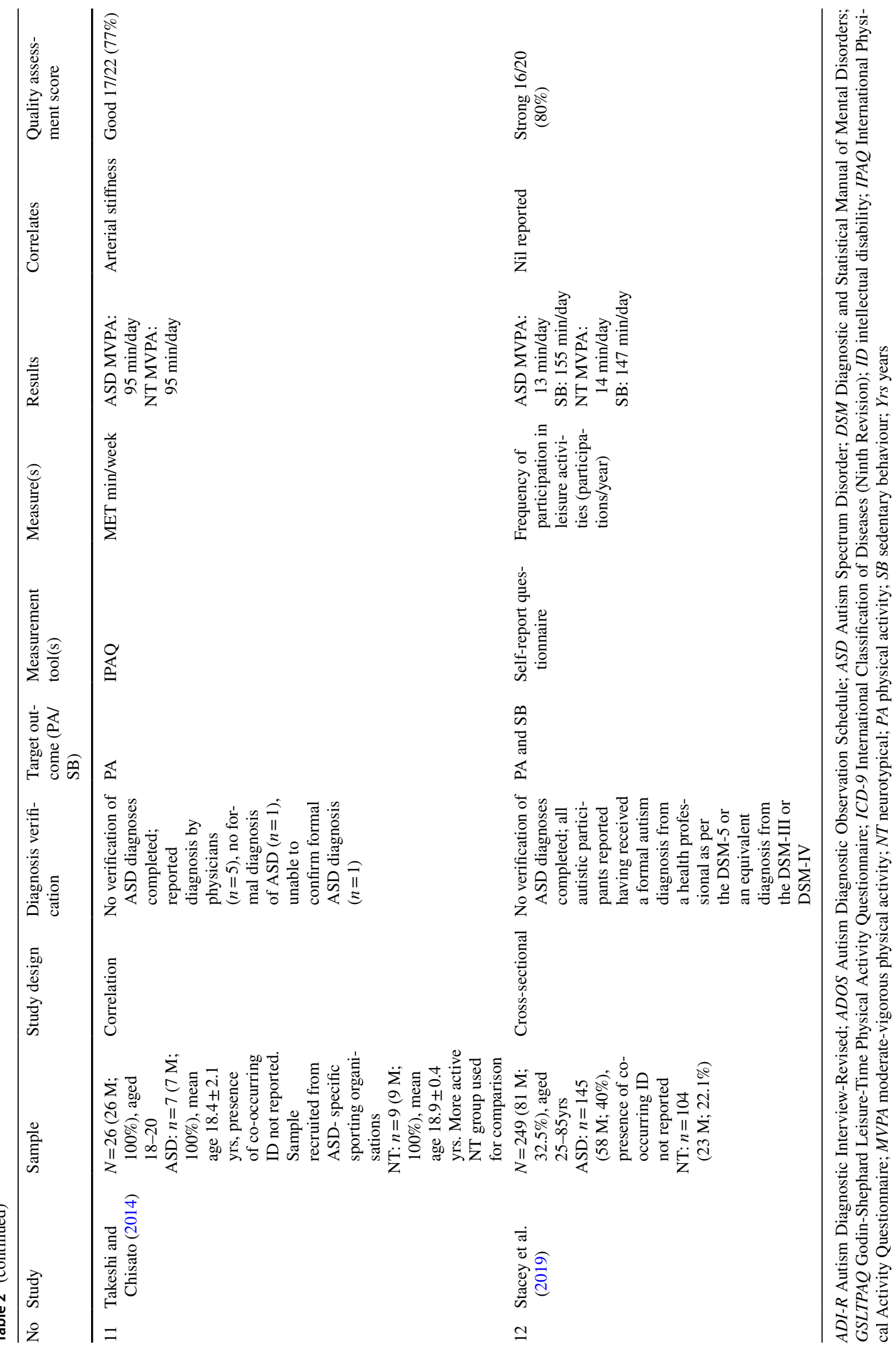


behaviour, the prevalence was reported as sedentary time (e.g. minutes/day) or time spent playing video games (e.g. hours/day, hours per week, days/week).

Prior to data synthesis and comparison, estimates of time spent in MVPA and sedentary behaviour were standardised to minutes per day to produce a comparable data set. The included studies were divided among two authors (SH, RT) to complete standardisation calculations. The data standardisation process required some assumptions be made in order to convert the available reported data into the form of MVPA and sedentary behaviour minutes per day. For a full explanation of the standardisation process and any assumptions made for each data format, see Appendix B. All calculations were initially completed manually on paper with the assistance of scientific calculators. All completed calculations were transcribed onto an electronic document by one author $(\mathrm{SH})$ and cross-checked by two authors ( $\mathrm{SH}$, $\mathrm{RT})$. Where discrepancies occurred, resolution was achieved through discussion. For full details of all completed calculations for each included study, see Appendix C. Final results of MVPA and sedentary behaviour minutes per day were rounded to the nearest whole number as per standard mathematical protocol.

\section{Results}

\section{Study Characteristics}

A total of 12 studies met the inclusion criteria for this review with six studies reporting physical activity (Benson et al., 2019; Hamm \& Yun, 2019; LaLonde et al., 2014; Li et al., 2019; Nastasi et al., 2020; Takeshi \& Chisato, 2014), two studies reporting sedentary behaviour (Engelhardt et al., 2017; Finke et al., 2018) and four studies including data on both physical activity and sedentary behaviour (BishopFitzpatrick et al., 2017; Garcia-Pastor et al., 2019; Jozkowski \& Cermak, 2020; Stacey et al., 2019) (Figure 1). Table 2 provides a summary of the characteristics of the included studies.

The average age of participants in the included studies was approximately 27 years (range $=18-85$ years). Of the 12 studies, one study used a sample comprising solely of males (Takeshi \& Chisato, 2014), and another included one single female participant (Finke et al., 2018). All remaining studies gathered data from a sample with a higher proportion of male compared to female participants (Garcia-Pastor et al., 2019; LaLonde et al., 2014; Li et al., 2019; Nastasi et al., 2020; Stacey et al., 2019). Within the included studies, six made use of a neurotypical control group for comparative purposes (Benson et al., 2019; Engelhardt et al., 2017; Hamm \& Yun, 2019; Jozkowski \& Cermak, 2019; Otsuki \& Ohashi, 2014; Stacey et al.,
2019). A range of measurement tools were used across the 12 studies, with seven studies using self-report measures (Benson et al., 2019; Bishop-Fitzpatrick et al., 2017; Engelhardt et al., 2017; Garcia-Pastor et al., 2019; Hamm \& Yun, 2019; Jozkowski \& Cermak, 2020; LaLonde et al., 2014; Li et al., 2019; Nastasi et al., 2020; Stacey et al., 2019), four studies using a measurement device (Nastasi et al., 2020; Pinder-Amaker, 2014) and one study using both self-report and measurement device (Benson et al., 2019). All studies included in Table 2 describe their research objective(s) in sufficient detail and are published in peer-reviewed journals. Sample sizes ranged from 4 to 320 participants, with one study obtaining data from one autistic adult participant (Nastasi et al., 2020). Of the 12 studies, four collected data from a sample of 10 participants or less (Finke et al., 2018; LaLonde et al., 2014; Li et al., 2019; Nastasi et al., 2020). Several sampling methods were used across the studies, with one recruiting autistic participants exclusively from ASD-specific sporting organisations (Takeshi \& Chisato, 2014).

\section{Autistic Adults' Physical Activity}

\section{Self-Report Findings}

Of the 12 studies, six reported data of time spent in physical activity obtained from a form of self-report measure $($ mean $=35 \mathrm{MVPA} \min /$ day, range $=13-95)($ Benson et al., 2019; Bishop-Fitzpatrick et al., 2017; Hamm \& Yun, 2019; Jozkowski \& Cermak, 2020; Stacey et al., 2019; Takeshi \& Chisato, 2014). Estimates of autistic adults' physical activity levels from questionnaires commonly used to measure physical activity in the general population (e.g. International Physical Activity Questionnaire, GSLTPAQ) were higher (mean $=54 \mathrm{MVPA} \mathrm{min} /$ day, range $=28-95$ ) than estimates from questionnaires tailored to meet specific study objectives and for use with autistic adults (mean $=16 \mathrm{MVPA}$ min/day, range $=$ 13-18) (Jozkowski \& Cermak, 2020; Stacey et al., 2019). Estimates obtained from interviews were more similar to the tailored questionnaire estimates (15 MVPA min/day) (Bishop-Fitzpatrick et al., 2017). In one study using both self- and proxy-report, the mother-reported value of daily physical activity was lower than that reported by the autistic adult participants themselves (mother-report mean = $8 \mathrm{MVPA} \mathrm{min} /$ day vs self-report mean $=15 \mathrm{MVPA} \mathrm{min} /$ day) (Bishop-Fitzpatrick et al., 2017). Overall, within the six studies measuring physical activity participation using self-report, three of the autistic adult samples would be deemed insufficiently active (i.e. not participate in $>150$ MVPA min/week) (Bishop-Fitzpatrick et al., 2017; Jozkowski \& Cermak, 2020; Stacey et al., 2019). 


\section{Objective Findings}

Within the included studies, five reported estimates of time spent in PA from measurement devices (mean $=52 \mathrm{MVPA}$ $\mathrm{min} /$ day, range $=16-73$ ) (Benson et al., 2019; Garcia-Pastor et al., 2019; LaLonde et al., 2014; Li et al., 2019; Nastasi et al., 2020). The two categories of these measurement devices used within these five studies were wearable activity trackers (i.e. Fitbit, Garmin) and research-grade accelerometers (i.e. Motionlogger and GT1M ActiGraph).

Within this review, three studies used wearable activity trackers to obtain data on autistic adults' PA ( mean $=68$ MVPA min/day, range $=61-73$ ) (LaLonde et al., 2014; Li et al., 2019; Nastasi et al., 2020). All three studies using wearable activity trackers for physical activity measurement scored less than 60\% when assessed with the Standard Quality Assessment Criteria and hence were classified to be of 'adequate' quality (Kmet et al., 2004; LaLonde et al., 2014; Lee et al., 2008; Li et al., 2019; Nastasi et al., 2020). All the participants in these three studies would be considered to participate in sufficient physical activity (i.e. participate in $>150 \mathrm{MVPA} \mathrm{min} /$ week).

Of the 12 studies, two used accelerometers to estimate autistic adults' time spent in physical activity (mean = 28 MVPA min/day, range $=16-40$ ) (Benson et al., 2019; Garcia-Pastor et al., 2019). These studies scored 77 (Benson et al., 2019) and 82\% (Garcia-Pastor et al., 2019) when assessed with the Standard Quality Assessment Criteria and hence were classified to be of 'good' and 'strong' quality, respectively (Kmet et al., 2004; Lee et al., 2008). Estimates of physical activity obtained from accelerometers were lower ( mean = $28 \mathrm{MVPA}$ min/day, range $=16-40)$ (Benson et al., 2019; Garcia-Pastor et al., 2019) compared to physical activity estimates from wearable activity trackers (mean $=68$ MVPA min/day, range $=61-73)$ (LaLonde et al., 2014; Li et al., 2019; Nastasi et al., 2020).

\section{Autistic Adults' Sedentary Behaviour}

\section{Self-Report Findings}

Within the included studies, five reported estimates of time spent in sedentary behaviour from a form of self-report measure (mean $=162$ sedentary behaviour $\mathrm{min} /$ day, range =72-266) (Bishop-Fitzpatrick et al., 2017; Engelhardt et al., 2017; Finke et al., 2018; Jozkowski \& Cermak, 2020; Stacey et al., 2019). Estimates of sedentary behaviour levels gathered from questionnaires were lower (mean $=158$ sedentary behaviour min/day, range $=150-168)($ Engelhardt et al., 2017; Jozkowski \& Cermak, 2020; Stacey et al., 2019) than estimates from in-person interviews (mean $=$ 169 sedentary behaviour $\mathrm{min} /$ day, range $=72-266$ ) (BishopFitzpatrick et al., 2017; Finke et al., 2018). In one study, mother-reported SB estimates were lower than that obtained from interviews with the autistic adult (mother-report mean $=37$ sedentary behaviour min/day vs self-report mean $=$ 72 sedentary behaviour min/day) (Bishop-Fitzpatrick et al., 2017).

\section{Objective Findings}

Out of the 12 studies, one reported device-derived estimates of autistic adults' time spent in sedentary behaviour obtained using the GITM accelerometer (1100 sedentary behaviour $\mathrm{min} /$ day) (Garcia-Pastor et al., 2019).

\section{Studies Including Neurotypical Adults}

A total of six studies included a neurotypical control group alongside autistic adult samples for comparison (Benson et al., 2019; Engelhardt et al., 2017; Hamm \& Yun, 2019; Jozkowski \& Cermak, 2020; Stacey et al., 2019; Takeshi \& Chisato, 2014). Another two studies included participants without ASD. However, due to the diagnosis of other neurological conditions (e.g. cognitive impairments, mental health conditions), they did not fit the criteria for being neurotypical participants (Li et al., 2019; Nastasi et al., 2020). All six studies, including neurotypical adult participants, relied on self-report for measurement of physical activity and/ or sedentary behaviour participation (Benson et al., 2019; Engelhardt et al., 2017; Hamm \& Yun, 2019; Jozkowski \& Cermak, 2020; Stacey et al., 2019; Takeshi \& Chisato, 2014). In addition to a self-report questionnaire, one study also obtained estimates regarding physical activity participation of autistic and neurotypical adults via accelerometry (Benson et al., 2019).

\section{Physical Activity Findings}

Of the six studies with autistic and neurotypical participants, five reported estimates of physical activity participation (ASD mean $=35$ MVPA min/day, range $=13-95 ;$ NT mean = 46 MVPA min/day, range $=14-95$ ) (Benson et al., 2019; Hamm \& Yun, 2019; Jozkowski \& Cermak, 2020; Stacey et al., 2019; Takeshi \& Chisato, 2014). Of these studies, autistic adults' level of physical activity participation was lower than the neurotypical adult sample in four of the studies (Benson et al., 2019; Hamm \& Yun, 2019; Jozkowski \& Cermak, 2020; Stacey et al., 2019). The difference in autistic adults' MVPA minutes per day ranged from 1 to 21 min less than that of the neurotypical adults (Benson et al., 2019; Stacey et al., 2019). The greatest difference in daily physical activity participation between groups was in the one study that used accelerometry for physical activity measurement (Benson et al., 2019). In one study, MVPA minutes per day 
were the same for autistic and neurotypical adults (Takeshi \& Chisato, 2014).

\section{Sedentary Behaviours Findings}

Of the six included studies with both autistic and neurotypical participants, three studies reported data about time spent in sedentary behaviour (ASD mean $=158$ sedentary behaviour $\mathrm{min} /$ day, range $=150-168$; neurotypical mean $=$ 85 sedentary behaviour $\mathrm{min} /$ day, range $=45-147$ ) (Engelhardt et al., 2017; Jozkowski \& Cermak, 2020; Stacey et al., 2019). Within all three of these studies, autistic adults' level of sedentary behaviour participation was reported to be higher than the neurotypical adults (Engelhardt et al., 2017; Jozkowski \& Cermak, 2020; Stacey et al., 2019). The difference in autistic adults' sedentary behaviour minutes per day ranged from 8 to 105 min greater than the neurotypical adults (Engelhardt et al., 2017; Jozkowski \& Cermak, 2020; Stacey et al., 2019).

\section{Correlates}

Within this review, eight studies reported correlates of physical activity, sedentary behaviour or both among autistic adults (Table 2) (Benson et al., 2019; Bishop-Fitzpatrick et al., 2017; Engelhardt et al., 2017; Finke et al., 2018; Garcia-Pastor et al., 2019; Hamm \& Yun, 2019; Jozkowski \& Cermak, 2020; Takeshi \& Chisato, 2014). A total of ten potential correlates were identified within the studies. The most commonly described correlate for time spent in physical activity and sedentary behaviour was day of the week (i.e. weekday vs weekend) (Benson et al., 2019; Engelhardt et al., 2017; Garcia-Pastor et al., 2019). In two studies, the day of the week was suggested to have no influence on autistic adults' physical activity and sedentary behaviour levels (Benson et al., 2019; Finke et al., 2018). Alternatively, the remaining study reported a larger number of hours per day spent in sedentary behaviour, specifically videogames, on weekends compared to weekdays for both autistic and neurotypical adults (Engelhardt et al., 2017).

Participant's fitness level was positively associated with levels of physical activity (Jozkowski \& Cermak, 2020). Sleep, health-related quality of life and social interaction were all also shown to be positively correlated with physical activity levels (Benson et al., 2019; Hamm \& Yun, 2019; Jozkowski \& Cermak, 2020). Conversely, obesity was negatively associated with autistic adults' level of physical activity, and older participants were observed to have lower levels of physical activity declined (Garcia-Pastor et al., 2019). In males, arterial stiffness was negatively associated with physical activity level (Takeshi \& Chisato, 2014). Additionally, levels of perceived stress were reported to be negatively associated with participants' physical activity and sedentary behaviour levels (Bishop-Fitzpatrick et al., 2017).

Higher levels of sedentary behaviour participation were reported to be positively associated with being of male gender compared to female (Finke et al., 2018). As participants aged, their sedentary behaviour participation was also found to increase (Garcia-Pastor et al., 2019). Furthermore, pathological game use symptoms were reported to have positive associations with time spent in sedentary behaviour (Engelhardt et al., 2017; Garcia-Pastor et al., 2019).

\section{Discussion}

This systematic review investigated what is currently known about autistic adults' physical activity and sedentary behaviour levels, along with associated correlates to these behaviours. Of the 2834 studies identified through database searching, only 12 met the inclusion criteria for analysis. Findings of this review highlight the scarcity of available evidence on the physical activity and sedentary behaviour of autistic adults. Additionally, various data collection methods were used within the primary studies, some of which may not be best suited for use within the autistic adult population. Of the six studies using self-report measures of physical activity, three of the autistic adult samples would be deemed to be insufficiently active (i.e. not participate in $>$ 150 MVPA min/week) (Bishop-Fitzpatrick et al., 2017; Jozkowski \& Cermak, 2020; Stacey et al., 2019). The results of one of the two studies using accelerometry identified that their sample as not meeting the physical activity guidelines (i.e. not participate in $>150 \mathrm{MVPA} \mathrm{min} /$ week) (Benson et al., 2019). These differing data collection methods also yielded inconsistent estimates of autistic adults' physical activity and sedentary behaviour participation. Therefore, estimates obtained from this review are not likely to be a true representation of autistic adults' physical activity and sedentary behaviour participation. This signals the need for additional studies using more accurate and reliable methods to quantify these behaviours.

The primary data collection method used by the studies included in this review was self-report. Self-report may be prone to inaccuracies regarding the frequency, duration and intensity of activities retrospectively recalled (Dyrstad et al., 2014). This can result in an overestimation of activity levels when compared to device-derived measures (Benson et al., 2019). Self-reporting accuracy limitations may be amplified among autistic adults due to their difficulties with social communication and interactions and/or potential co-occurring intellectual disability (American Psychiatric Association, 2013); Benson et al., 2019). In addition, most questionnaires only allow for reporting of time spent in sedentary leisure activities (e.g. videogaming, watching 
television). This does not take into consideration time spent sedentary during productive activities (e.g. office work, university lectures), which are likely to be a large proportion of autistic adults' daily activity. So evidenced by the wide range of estimates obtained, relying solely on self-report measures to estimate autistic adults' physical activity and sedentary behaviour may not be a reliable data collection method.

Measurement devices, such as accelerometers or wearable activity trackers, are not reliant on participants' ability to understand questions and/or respectively recall activity. Therefore, measurement devices are more likely to capture accurate estimates of physical activity and sedentary behaviour throughout the day. Accelerometry has been validated across multiple populations and is currently viewed as the 'gold standard' measurement of activity levels (Sylvia et al., 2014; Verceles \& Hager, 2015). In the current review, accelerometers were only used in two studies (Benson et al., 2019; Garcia-Pastor et al., 2019), and one of these did not separate sleep time from daytime sedentary behaviour in their results (Benson et al., 2019). This makes the interpretation of sedentary behaviour estimates difficult and may have skewed results. Wearable activity trackers may be useful in studies wanting to objectively measure time spent in autistic adults' physical activity and sedentary behaviour, due to their lower cost, accessibility, and low burden of wear (Semanik et al., 2020). Wearable activity trackers were used in three studies. However, their small sample sizes and low ecological validity should also be considered in the interpretation of results (LaLonde et al., 2014; Li et al., 2019; Nastasi et al., 2020). Due to the limited number of high-quality studies using measurement devices, the proportion of the autistic adult population meeting the recommended levels of physical activity is unknown.

The quantification of physical activity and sedentary behaviour has been inconsistent between neurotypical adults and autistic adults. The ability to accurately quantify physical activity and sedentary behaviour is important due to their association with chronic health conditions and mental wellbeing (Kokkinos, 2012; World Health Organization, 2017). The secondary sequelae of insufficient physical activity may be exaggerated for autistic adults due to a greater incidence of poor health outcomes, such as lower life expectancy and higher rates of mental health conditions compared to neurotypical adults (Hirvikoski et al., 2016; van Heijst \& Geurts, 2015). Current research has also shown that physical activity participation can provide additional benefits specific to the autistic adult population, such as reduced stereotypic behaviours, anxiety, and stress levels, as well as providing valuable opportunities for social interaction (Lang et al., 2010). Factors reported to be associated with autistic adults' physical activity and sedentary behaviour participation were limited within the included studies yet provide initial insights for future studies to consider.
Correlates to autistic adults' physical activity and sedentary behaviour were reported in eight studies, with 10 potential correlates defined. Day of the week was the only correlate to be reported across multiple studies, yet findings were inconsistent (Benson et al., 2019; Engelhardt et al., 2017; Garcia-Pastor et al., 2019). In one study, autistic adults were demonstrated to spend greater time in sedentary behaviour on weekends compared to weekdays (Engelhardt et al., 2017), which is consistent with findings with prior research on neurotypical adults (Marshall et al., 2015). Additionally, it can be presumed that individuals would also participate in lower levels of physical activity on weekends compared to weekdays (Chaput et al., 2014). This is similar to what has been shown in autistic adolescents, whereby time spent in physical activity was significantly lower on weekends compared to weekdays (Stanish et al., 2017). These differences may be attributed to having more free time available on weekends than weekdays and providing a basis for research to increase weekend physical activity participation in the autistic population. However, due to scarce and inconsistent reporting of the impact that the day of the week has on autistic adults' activity levels, it is unclear how this can be targeted by interventions until clearer measurements of these behaviours on days of the week are obtained. Furthermore, studies on autistic children and neurotypical adults report levels of physical activity participation to be negatively associated with increasing age and poorer health, and positively associated with social interaction and being of male gender (Bennie et al., 2016; Healy \& Garcia, 2019; Jones et al., 2017; Pan et al., 2011). These correlates show similarities to those reported across the studies of this review and may also apply to autistic adults.

The interpretations of this review should be considered in the context of the limitations present within the primary studies. Such limitations included the poor quality of reported data and questionable reliability and accuracy of data collection methods. Due to the differing nature of the primary studies, a series of assumptions had to be made to allow for standardisation (Appendix B) and, thus, limit the accuracy of numerical values derived. Furthermore, the potential occurrence of common methodological flaws, such as inaccuracies in assessing the quality of included studies, cannot be ruled out (Garg et al., 2008). Biases may have also occurred as there is inevitably subjectivity in decisionmaking to determine study eligibility. Finally, the authors manually extracted data from the studies and did not contact primary researchers for verification of accuracy or to attain additional research information (e.g. missing/unreported data). This may have impacted the reliability of reported results and subsequent interpretations, further limiting extrapolation of findings.

Future research examining autistic adults' physical activity and sedentary behaviour participation should use more 
accurate and reliable methods to capture the information. In particular, measurement devices are recommended over self-report measures for this population as they eliminate potential inaccuracies due to the possibility of autistic adults having difficulty interpreting questions. Studies including neurotypical control groups and large samples representative of the autistic adult population are also warranted. Future studies should also examine a range of potential correlates to allow for the directionality and relative strength of associations with physical activity and sedentary behaviour to be examined. This may assist in the development of tailored interventions to promote physical activity in the autistic adult population.

Currently, it is not possible to accurately estimate autistic adults' physical activity and sedentary behaviour levels due to the poor quality of the current literature and the limited number of studies. However, based on the available evidence, it appears that autistic adults may be less physically active and more sedentary than neurotypical adults and that correlates that influence these behaviours may exist. Hence, this population may be at greater risk of numerous physical and mental health consequences. Accurate estimates of autistic adults' physical activity and sedentary behaviour are urgently needed in order to determine the true extent of inactivity and the proportion of the autistic adult population participating in sufficient physical activity.

Supplementary Information The online version contains supplementary material available at https://doi.org/10.1007/s40489-022-00305-x.

Acknowledgements This work would be impossible without the contributions of the autistic individuals across the world. We are grateful to the Curtin School of Allied Health for the support of the research.

Funding Open Access funding enabled and organized by CAUL and its Member Institutions.

\section{Declarations}

Conflict of Interest All authors declare no competing interests.

Open Access This article is licensed under a Creative Commons Attribution 4.0 International License, which permits use, sharing, adaptation, distribution and reproduction in any medium or format, as long as you give appropriate credit to the original author(s) and the source, provide a link to the Creative Commons licence, and indicate if changes were made. The images or other third party material in this article are included in the article's Creative Commons licence, unless indicated otherwise in a credit line to the material. If material is not included in the article's Creative Commons licence and your intended use is not permitted by statutory regulation or exceeds the permitted use, you will need to obtain permission directly from the copyright holder. To view a copy of this licence, visit http://creativecommons.org/licenses/by/4.0/.

\section{References}

Australian Bureau of Statistics. (2019). National health survey: First results, 2017-18 (No. 4364.055.001). https://www.abs.gov.au/ ausstats/abs@.nsf/Lookup/by\%20Subject/4364.0.55.001 201718 Main\%20Features Physical\%20activity 115

Department of Health. (2019). Australia's physical activity and sedentary behaviour guidelines and the Australian 24-hour movement guidelines. https://www1.health.gov.au/internet/main/publishing. nsf/Content/health-pubhlth-strateg-phys-act-guidelines

Bennie, J. A., Pedisic, Z., van Uffelen, J. G. Z., Gale, J., Banting, L. K., Vergeer, I., Stamatakis, E., Bauman, A. E., \& Biddle, S. J. H. (2016). The descriptive epidemiology of total physical activity, muscle-strengthening exercises and sedentary behaviour among Australian adults - results from the National Nutrition and Physical Activity Survey. BMC Public Health, 16(1), 73. https://doi. org/10.1186/s12889-016-2736-3

Benson, S., Bender, A. M., Wickenheiser, H., Naylor, A., Clarke, M., Samuels, C. H., \& Werthner, P. (2019). Differences in sleep patterns, sleepiness, and physical activity levels between young adults with autism spectrum disorder and typically developing controls. Developmental Neurorehabilitation, 22(3), 164-173 (2019). https://doi.org/10.1080/17518423.2018.1501777

Bishop-Fitzpatrick, L., Smith DaWalt, L., Greenberg, J. S., \& Mailick, M. R. (2017). Participation in recreational activities buffers the impact of perceived stress on quality of life in adults with autism spectrum disorder. Autism Research, 10(5), 973-982. https://doi.org/10.1002/aur.1753

Chaput, J.-P., Carson, V., Gray, C. E., \& Tremblay, M. S. (2014). Importance of all movement behaviors in a 24 hour period for overall health. International Journal of Environmental Research and Public Health, 11(12), 12575-12581. https://www.mdpi. com/1660-4601/11/12/12575

Dyrstad, S. M., Hansen, B. H., Holme, I. M., \& Anderssen, S. A. (2014). Comparison of self-reported versus accelerometermeasured physical activity. Medicine \& Science in Sports \& Exercise, 46(1), 99-106. https://doi.org/10.1249/MSS.0b013 e3182a0595f

Engelhardt, C. R., Mazurek, M. O., \& Hilgard, J. (2017). Pathological game use in adults with and without autism spectrum disorder. PeerJ, 5, e3393. https://doi.org/10.7717/peerj.3393

Finke, E. H., Hickerson, B. D., \& Kremkow, J. M. D. (2018). \&\#x201c;To be quite honest, if it wasn't for videogames i wouldn't have a social life at All\&\#x201d;: Motivations of young adults with autism spectrum disorder for playing videogames as leisure. American Journal of Speech-Language Pathology, 27(2), 672-689 (2018). doi:https://doi.org/10.1044/2017_AJSLP-17-0073

Garcia-Pastor, T., Salinero, J. J., Theirs, C. I., \& Ruiz-Vicente, D. (2019). Obesity status and physical activity level in children and adults with autism spectrum disorders: A pilot study. Journal of Autism Developmental Disorders, 49(1), 165-172. https://doi.org/ 10.1007/s10803-018-3692-9

García-Villamisar, D. A., \& Dattilo, J. (2010). Effects of a leisure programme on quality of life and stress of individuals with ASD. Journal of Intellectual Disability Research, 54(7), 611-619. https://doi.org/10.1111/j.1365-2788.2010.01289.x

Garg, A. X., Hackam, D., \& Tonelli, M. (2008). Systematic review and meta-analysis: When one study is just not enough. Clinical Journal of the American Society of Nephrology, 3(1), 253-260. https://doi.org/10.2215/cjn.01430307

Hamm, J., \& Yun, J. (2019). Influence of physical activity on the health-related quality of life of young adults with and without autism spectrum disorder. Disability and Rehabilitation, 41(7), 763-769. https://doi.org/10.1080/09638288.2017.1408708 
Healy, S., \& Garcia, J. M. (2019). Psychosocial correlates of physical activity participation and screen-time in typically developing children and children on the autism spectrum. Journal of Developmental and Physical Disabilities, 31(3), 313-328. https://doi. org/10.1007/s10882-018-9642-9

Healy, S., Aigner, C. J., Haegele, J. A., \& Patterson, F. (2019). Meeting the 24-hr movement guidelines: An update on US youth with autism spectrum disorder from the 2016 National Survey of Children's Health. Autism Research, 12(6), 941-951. https://doi.org/ 10.1002/aur.2095

Hillier, A., Buckingham, A., \& Schena, D. (2020). Physical activity among adults with autism: Participation, attitudes, and barriers. Perceptual and Motor Skills, 127(5), 874-890, https://doi.org/10. 1177/0031512520927560

Hirvikoski, T., Mittendorfer-Rutz, E., Boman, M., Larsson, H., Lichtenstein, P., \& Bölte, S. (2016). Premature mortality in autism spectrum disorder. British Journal of Psychiatry, 208(3), 232-238. https://doi.org/10.1192/bjp.bp.114.160192

Howlin, P., Moss, P., Savage, S., Bolton, P., \& Rutter, M. (2015). Outcomes in adult life among siblings of individuals with autism. Journal of Autism and Developmental Disorders, 45(3), 707-718. https://doi.org/10.1007/s10803-014-2224-5

Jones, R. A., Downing, K., Rinehart, N. J., Barnett, L. M., May, T., McGillivray, J. A., Papadopoulos, N. V., Skouteris, H., Timperio, A., \& Hinkley, T. (2017). Physical activity, sedentary behavior and their correlates in children with autism spectrum disorder: A systematic review. PLoS ONE, 12(2), e0172482. https://doi.org/ 10.1371/journal.pone.0172482

Jozkowski, A. C., \& Cermak, S. A. (c). (2019). Moderating effect of social interaction on enjoyment and perception of physical activity in young adults with autism spectrum disorders. International Journal of Developmental Disabilities, 66(3), 222-234. https:// doi.org/10.1080/20473869.2019.1567091

Kmet, L., Lee, R., \& Cook, L. (2004). Standard quality assessment criteria for evaluating primary research papers from a variety of fields. https://www.ihe.ca/advanced-search/standard-quality-asses sment-criteria-for-evaluating-primary-research-papers-from-avariety-of-fields

Knüppel, A., Telléus, G. K., Jakobsen, H., \& Lauritsen, M. B. (2019). Characteristics of young adults with autism spectrum disorder performing different daytime activities. Journal of Autism and Developmental Disorders, 49(2), 542-555. https://doi.org/10. 1007/s10803-018-3730-7

Kokkinos, P. (2012). Physical activity, health benefits, and mortality risk. ISRN Cardiology, 2012, 718789. https://doi.org/10.5402/ 2012/718789

LaLonde, K. B., MacNeill, B. R., Eversole, L. W., Ragotzy, S. P., \& Poling, A. (2014). Increasing physical activity in young adults with autism spectrum disorders. Research in Autism Spectrum Disorders, 8(12), 1679-1684. https://doi.org/10.1016/j.rasd.2014. 09.001

Lang, R., Koegel, L. K., Ashbaugh, K., Regester, A., Ence, W., \& Smith, W. (2010). Physical exercise and individuals with autism spectrum disorders: A systematic review. Research in Autism Spectrum Disorders, 4(4), 565-576 (2010). doi:https://doi.org/ 10.1016/j.rasd.2010.01.006

Lear, S. A., Hu, W., Rangarajan, S., Gasevic, D., Leong, D., Iqbal, R., Casanova, A., Swaminathan, S., Anjana, R. M., Kumar, R., Rosengren, A., Wei, L., Yang, W., Chuangshi, W., Huaxing, L., Nair, S., Diaz, R., Swidon, H., Gupta, R., Mohammadifard, N., Lopez-Jaramillo, P., Oguz, A., Zatonska, K., Seron, P., Avezum, A., Poirier, P., Teo, K., \& Yusuf, S. (2017). The effect of physical activity on mortality and cardiovascular disease in 130000 people from 17 high-income, middle-income, and low-income countries: the PURE study. The Lancet, 390(10113), 2643-2654 (2017). doi:https://doi.org/10.1016/S0140-6736(17)31634-3
Lee, L., Packer, T. L., Tang, S. H., \& Girdler, S.. Self-management education programs for age-related macular degeneration: A systematic review. Australasian Journal on Ageing, 27(4), 170-176. https://doi.org/10.1111/j.1741-6612.2008.00298.x

Li, A., Curiel, H., Ragotzy, S. P., \& Poling, A. (2019). Using a lottery to promote physical activity by young adults with developmental disabilities. Behavior Analysis in Practice, 12(3), 612-616. https://doi.org/10.1007/s40617-018-00292-8

MacDonald, M., Esposito, P., \& Ulrich, D. (2011). The physical activity patterns of children with autism. BMC Research Notes, 4(1), 422 https://doi.org/10.1186/1756-0500-4-422

Marshall, S., Kerr, J., Carlson, J., Cadmus-Bertram, L., Patterson, R., Wasilenko, K., Crist, K., Rosenberg, D., \& Natarajan, L. (2015). Patterns of weekday and weekend sedentary behavior among older adults. Journal of Aging and Physical Activity, 23(4), 534. https:// doi.org/10.1123/japa.2013-0208

Menear, K. S., \& Neumeier, W. H. (2015). Promoting physical activity for students with autism spectrum disorder: Barriers, benefits, and strategies for success. Journal of Physical Education, Recreation \& Dance, 86(3), 43-48. https://doi.org/10.1080/07303084.2014. 998395

Moher, D., Liberati, A., Tetzlaff, J., \& Altman, D. G. (2009). Preferred reporting items for systematic reviews and meta-analyses: The PRISMA statement. BMJ, 339, b2535. https://doi.org/10.1136/ bmj.b2535

Must, A., Phillips, S. M., Curtin, C., Anderson, S. E., Maslin, M., Lividini, K., \& Bandini, L. G. (2014). Comparison of sedentary behaviors between children with autism spectrum disorders and typically developing children. Autism, 18(4), 376-384. https://doi. org/10.1177/1362361313479039

Nastasi, J. A., Sheppard, R. D., \& Raiff, B. R. (2020). Token-economybased contingency management increases daily steps in adults with developmental disabilities. Behavioral Interventions, 35(2), 315-324. https://doi.org/10.1002/bin.1711

National Institute for Health Research. (2020). Welcome to prospero International prospective register of systematic reviews. https:// www.crd.york.ac.uk/prospero

Nichols, C., Block, M. E., Bishop, J. C., \& McIntire, B. (2018). Physical activity in young adults with autism spectrum disorder: Parental perceptions of barriers and facilitators. Autism, 23(6), 1398-1407. https://doi.org/10.1177/1362361318810221

Pan, C.-Y., Tsai, C.-L., \& Hsieh, K.-W. (2011). Physical activity correlates for children with autism spectrum disorders in middle school physical education. Research Quarterly for Exercise and Sport, 82(3), 491-498. https://doi.org/10.1080/02701367.2011. 10599782

Pinder-Amaker, S. (2014). Identifying the unmet needs of college students on the autism spectrum. Harvard Review of Psychiatry, 22(2), 125-137. https://doi.org/10.1097/hrp.0000000000000032

American Psychiatric Association. (2013). The diagnostic and statistical manual of mental disorders: DSM 5. American Psychiatric Publishing.

Semanik, P., Lee, J., Pellegrini, C. A., Song, J., Dunlop, D. D., \& Chang, R. W. (2020). Comparison of physical activity measures derived from the fitbit flex and the ActiGraph GT3X+ in an employee population with chronic knee symptoms. ACR Open Rheumatology, 2(1), 48-52. https://doi.org/10.1002/acr2.11099

Simpson, K., Adams, D., Bruck, S., \& Keen, D. (2019). Investigating the participation of children on the autism spectrum across home, school, and community: A longitudinal study. Child: Care, Health and Development, 45(5), 681-687. https://doi.org/10.1111/cch. 12679

Stacey, T.-L., Froude, E. H., Trollor, J., \& Foley, K.-R. (2019). Leisure participation and satisfaction in autistic adults and neurotypical adults. Autism, 23(4), 993-1004. https://doi.org/10.1177/13623 61318791275 
Stanish, H. I., Curtin, C., Must, A., Phillips, S., Maslin, M., \& Bandini, L. G.. Physical activity levels, frequency, and type among adolescents with and without autism spectrum disorder. Journal of Autism and Developmental Disorders, 47(3), 785-794. https:// doi.org/10.1007/s10803-016-3001-4

Strath, S. J., Pfeiffer, K. A., \& Whitt-Glover, M. C. (2012). Accelerometer use with children, older adults, and adults with functional limitations. Medicine \& Science in Sports \& Exercise, 44(1S), S77-S85. https://doi.org/10.1249/MSS.0b013e3182399eb1

Sylvia, L. G., Bernstein, E. E., Hubbard, J. L., Keating, L., \& Anderson, E. J. (2014). Practical guide to measuring physical activity. Journal of the Academy of Nutrition and Dietetics, 114(2), 199-208. https://doi.org/10.1016/j.jand.2013.09.018

Takeshi, O., \& Chisato, O. (2014). Participation in physical activity and arterial stiffness in males with autism spectrum disorder. Artery Research, 8(3), 110-114. https://doi.org/10.1016/j.artres.2014. 04.001

Tanguay, P. E., \& Lohr, D. W. (2015). Autism spectrum disorders. In M. K. Duncan (Ed.), Dulcan's Textbook of Child and Adolescent Psychiatry (2nd ed., pp. 83-94). https://doi.org/10.1176/appi. books.9781615370306.md08

Tobin, M. C., Drager, K. D. R., \& Richardson, L. F. (2014). A systematic review of social participation for adults with autism spectrum disorders: Support, social functioning, and quality of life.
Research in Autism Spectrum Disorders, 8(3), 214-229. https:// doi.org/10.1016/j.rasd.2013.12.002

van Heijst, B. F., \& Geurts, H. M. (2015). Quality of life in autism across the lifespan: A meta-analysis. Autism, 19(2), 158-167. https://doi.org/10.1177/1362361313517053

Verceles, A. C., \& Hager, E. R. (2015). Use of accelerometry to monitor physical activity in critically ill subjects: A systematic review. Respiratory Care, 60(9), 1330-1336. https://doi.org/10.4187/ respcare. 03677

World Health Organization. (2017). Physical activity. https://www. who.int/dietphysicalactivity/pa/en/

Yano, S., Koohsari, M. J., Shibata, A., Ishii, K., Mavoa, S., \& Oka, K. (2019). Assessing physical activity and sedentary behavior under free-living conditions: Comparison of active style Pro HJA-350IT and ActiGraphTM GT3X+. International journal of environmental research and public health, 16(17), 3065 . https://www.mdpi. com/1660-4601/16/17/3065

Publisher's Note Springer Nature remains neutral with regard to jurisdictional claims in published maps and institutional affiliations. 